\title{
Logarithmic Divergence Measure for Fuzzy Matrix and Application
}

\author{
Alka Rani ${ }^{1, *}$, Omdutt Sharma ${ }^{2,+}$, Priti Gupta ${ }^{1, \#}$ \\ ${ }^{1}$ Department of Statistics, M.D. University, Rohtak 124001, India \\ ${ }^{2}$ Department of Mathematics, P.D.M. University, Bahadurgarh 124507, India. \\ *alka1singla@gmail.com, ${ }^{+}$omdutt86@gmail.com,${ }^{\text {ppriti mdu@yahoo.in }}$
}

\begin{abstract}
This paper introduces a new divergence measure for a fuzzy matrix with proof of its validity. In addition, the properties are proved for the new fuzzy divergence measure. A method to solve decision making problem is developed by using the proposed fuzzy divergence measure. Finally, the application of this fuzzy divergence measure to decision making is shown using real-life example.
\end{abstract}

Keywords: Fuzzy Sets, Fuzzy Matrices, Divergence Measures, Decision Making Problem.

\section{INTRODUCTION}

A general problem in all fields like Mathematics, Science and Engineering is to distinguish two probability distributions $P=$ $\left(p_{1}, p_{2}, \ldots p_{n}\right)$ and $Q=\left(q, q_{2}, \ldots q_{n}\right)$. Measurement of this distance in Information theory is called divergence measure. Information theoretic measures (entropy, similarity measures and divergence measures) are consequential tools to manage the precarious information and successfully applied in different directions. Fuzziness or degree of diceyness is measured by the tool called Entropy. De Luca and Termini (1972) commenced the fuzzy entropy measures to define the uncertainty between fuzzy sets. Subsequently, entropy measures of fuzzy sets received great curiosity from researchers in different areas like analysis of contingency table by Gokhle and Kullback(1978), in approximation of probability distributions by Chao and Liu
(1968), Kazakos and Cotsidas (1980), Lin and Wong (1988), in signal processing Kadota ansd Shepp (1967) and in pattern recognition by Ben (1978), Chen (1973) and in many other areas. To measure the distinct information between fuzzy sets, essentially Bhandari and Pal (1993) explored fuzzy divergence measure. In due course, various divergence measures developed and modified by kapur (1997), Parkash (2000), Rosenfeld (1985) for fuzzy sets. Bhandari and Pal(1993) introduced divergence measure for fuzzy sets, defined $D\left(A, A^{\text {near }}\right)$ ratio to $D\left(A, A^{\text {far }}\right)$ an ambiguity measure and $\alpha$-order fuzzy entropy using Renyi's $\alpha$ order probabilistic entropy. Afterwards, various scholars have consciousness on divergence measure for fuzzy sets. Corresponding to information measure of Bhandari and Pal (1993), Fan and Xie (1999) introduce exponential fuzzy divergence measure based on operations. They also studied its relation with divergence measure introduced by Bhandari and Pal (1993). Montes et al. (2002) studied the special classes of divergence measures and used the link between fuzzy and probabilistic uncertainty. Bajaj and Hooda (2010) generalized the measure of fuzzy directed divergence. Fuzzy directed divergence measure has wide range of applications in different areas. Sharma et al. (2020) proposed a non-probabilistic divergence measure for fuzzy matrices and applied the same in decision making problem and in feature selection problem. Rani et al. (2020) studied an information 
measure and found application of fuzzy soft matrices. Vlachos and Sergiadis (2007) introduced the divergence measure for IFSs, studied the relationship between divergence measure and entropy measures and found the application in image segmentation, pattern recognition and medical diagnosis.

The remaining part of paper is organized as follows. Section 2 is devoted to introduce some conventional concepts, and notions related to fuzzy set theory and fuzzy matrix theory. In section 3, we proposed a new fuzzy divergence measure for fuzzy matrix corresponding to Bhandari and Pal (1993). Section 4 provides more dignified properties of the proposed measure in form of theorems. It is followed by the applications of the proposed divergence measure for the IFM to multi-criteria decision making (MCDM) and multi-attribute decision making (MADM) with a numerical example in section 5. Finally, some concluding remarks are drawn in section 6.

\section{PRELIMINARIES}

This section is completely devoted to explain some basic concepts and the assumptions of fuzzy set theory and fuzzy matrix theory. Then, we recall axiomatic definition of fuzzy divergence measure.

A. Fuzzy set:

(Rani et al. (2020)) The linguistic values of the alternatives assessment are usually represented by fuzzy sets to deal with uncertainty of real -world problems. Fuzzy sets are the sets having degree of membership as an element of the set. Zadeh (1965) acquainted the fuzzy sets as the expansion of the crisp sets.

Definition: (2020) A fuzzy set ḿ(ỳ) on Ủ is defined by a membership function ḿ(ỳ) : Ủ $\rightarrow[0,1]$. For ỳ $\in$ Ủ, ḿ (ỳ) the membership function denotes the degree to which ỳ belongs to fuzzy set m.

\section{B. Fuzzy Matrix Theory:}

The basic idea behind the fuzzy matrix theory is very elementary and an easily applicable in all types of circumstances. The algorithms and algebra of fuzzy matrix theory are applicable for data related problems. Social scientists apply this approach to analyze interactions between attributes and to analyze other analytical tools.

Definition: Fuzzy matrix (2020): A fuzzy matrix $A$ of order $m \times n$ is defined as $A=$ $\left[<a_{i j},>\right]_{m \times n}$ where $a_{i j}$ is the membership value of the element $a_{i j}$ in $A$. We may write $A$ as $A=\left[a_{i j}\right]_{m \times n}$. For example,

$$
A=\left[\begin{array}{lll}
0.1 & 0.3 & 0.4 \\
0.7 & 0.4 & 0.5 \\
0.2 & 0.1 & 0.9
\end{array}\right]
$$

is $3 \times 3$ fuzzy matrix.

Definition: Let $A=\left[a_{i j}\right] \in[F(\mathrm{M})]_{m \times n}$, If $m \neq n$, then $A$ is called a fuzzy rectangular matrix.

Definition: Let $A=\left[a_{i j}\right] \in[F(\mathrm{M})]_{m \times n}$, If $m=n$, then $A$ is called a fuzzy square matrix.

Definition: Let $A=\left[a_{i j}\right] \in[F(\mathrm{M})]_{m \times n}$, If $n=1$, then $A$ is called a fuzzy column matrix. For example,

$$
A=\left[\begin{array}{c}
0.2 \\
07 \\
0.5
\end{array}\right]
$$

is $3 \times 1$ column fuzzy matrix.

Definition: Let $\mathrm{A}=\left[a_{i j}\right] \in[F(\mathrm{M})]_{m \times n}$, If $m=1$, then $A$ is called a fuzzy row matrix. For example,

$$
A=\left[\begin{array}{lll}
0.1 & 0.5 & 0.8
\end{array}\right]
$$

is $1 \times 3$ row matrix.

\section{Operations on Two Fuzzy Matrices}

Here we performed some operation on fuzzy matrices. Two fuzzy matrices $A$ and $B$ of order $3 \times 3$ are taken for further operations as follows. 


$$
\begin{aligned}
& =\left[\begin{array}{lll}
0.7 & 0.5 & 0.2 \\
0.3 & 0.2 & 0.1 \\
0.3 & 0.7 & 0.6
\end{array}{ }^{A}{ }^{B}\right. \\
& =\left[\begin{array}{lll}
0.2 & 0.3 & 0.1 \\
0.5 & 0.2 & 0.6 \\
0.8 & 0.7 & 0.2
\end{array}\right]^{B}
\end{aligned}
$$

\section{Union (Addition) Operation of Two Fuzzy Matrices}

Definition (2020): Let $A=\left[a_{i j}\right], B=$ $\left[b_{i j}\right] \in[F(\mathrm{M})]_{m \times n}$. Then Union of fuzzy matrix $A, B$ is defined by $\left(A_{m \times n} \cup\right.$ $\left.B_{m \times n}\right)=C_{m \times n}=\left[c_{i j}\right]_{m \times n}$, where $c_{i j}=$ $\max \left(a_{i j}, b_{i j}\right)$ for all $i$ and $j$.

Example: The union operation of two matrices given by (2.2.1) and (2.2.2) is

$$
\left(A_{3 \times 3} \cup B_{3 \times 3}\right)=C_{3 \times 3}=\left[\begin{array}{lll}
0.7 & 0.5 & 0.2 \\
0.5 & 0.2 & 0.6 \\
0.8 & 0.7 & 0.6
\end{array}\right]
$$

Intersection Operation of Two Fuzzy Matrices

Definition (2020): Let $A=\left[a_{i j}\right], B=$ $\left[b_{i j}\right] \in[F(\mathrm{M})]_{m \times n}$. Then intersection of $A, B$ is defined by $\left(A_{m \times n} \cap B_{m \times n}\right)=$ $C_{m \times n}=\left[c_{i j}\right]_{m \times n}$, where $c_{i j}=\min \left(a_{i j}, b_{i j}\right)$ for all $i$ and $j$.

Example: The minimum operation of two matrices given by (2.2.1) and (2.2.2) is

$$
\left(A_{3 \times 3} \cap B_{3 \times 3}\right)=C_{3 \times 3}=\left[\begin{array}{lll}
0.2 & 0.3 & 0.1 \\
0.3 & 0.2 & 0.1 \\
0.3 & 0.7 & 0.2
\end{array}\right]
$$

Some researchers used suprimum and infimum operation as an analogous to our usual maximum and minimum.

Minimum-Maximum Operation of Two Fuzzy Matrices

Definition: Let $A=\left[a_{i j}\right] \in$ $[F(\mathrm{M})]_{m \times n} \& B=\left[b_{j i}\right] \in[\mathrm{F}(\mathrm{M})]_{n \times m}$.

Then Min-Max operation of $A, B$ is defined by $\quad \min -\max \left(A_{m \times n}, B_{m \times n}\right)=C_{m \times m}=$ $\left[c_{i j}\right]_{m \times m}, \quad$ where $\quad c_{i j}=$ $\min \left\{\max \left[\left(a_{i j}, b_{j i}\right)\right.\right.$ for $j=$ 1 to $n]$ f for $i=1$ to $m$.
Conjugate (Complement) of Fuzzy Matrix Definition (2020): Let $A=\left[a_{i j}\right], B=$ $\left[b_{i j}\right] \in[\mathrm{F}(\mathrm{M})]_{m \times n}$, then $A$ is conjugate (complement) of $B$ denoted by $B^{c}=A=$ $\left[a_{i j}\right]$, where $a_{i j}=1-b_{i j}$ for all $i$ and $j$.

Example: The conjugate (complement) operation of matrix given by (2.2.1) is

$$
A^{c}=B=\left[\begin{array}{lll}
0.3 & 0.5 & 0.8 \\
0.7 & 0.8 & 0.9 \\
0.7 & 0.3 & 0.4
\end{array}\right]
$$

Maximum-Minimum Operation of Two Fuzzy Matrices

Definition (2020): Let $A=\left[a_{i j}\right] \in$ $[F(\mathrm{M})]_{m \times n} \& B=\left[b_{j i}\right] \in[\mathrm{F}(\mathrm{M})]_{n \times m}$

Then Max-Min operation of $A, B$ is defined by $\max -\min \left(A_{m \times n}, B_{n \times m}\right)=C_{m \times m}=$ $\left[c_{i j}\right]_{m \times m}, \quad$ where $\quad c_{i j}=$ $\max \left\{\min \left[\left(a_{i j}, b_{j i}\right)\right.\right.$ for $j=$ 1 to $n]\}$ for $i=1$ to $m$.

Example: The max-min operation of two matrices given by (2.2.1) and (2.2.2) is

$$
\begin{gathered}
\max -\min \left(A_{3 \times 3}, B_{3 \times 3}\right)=C_{3 \times 3} \\
=\left[\begin{array}{lll}
0.5 & 0.3 & 0.5 \\
0.2 & 0.3 & 0.2 \\
0.6 & 0.6 & 0.6
\end{array}\right]
\end{gathered}
$$

where

$$
\begin{aligned}
& a_{11} \\
& =\max \{\min (0.7,0.2), \min (0.5,0.5), \min (0.2,0.8)\} \\
& =\max \{0.2,0.5,0.2\}=0.5 \\
& a_{12} \\
& =\max \{\min (0.7,0.3), \min (0.5,0.2), \min (0.2,0.7)\} \\
& =\max \{0.3,0.2,0.2\}=0.3 . \\
& \text { and so on }
\end{aligned}
$$

Example: The min-max operation of two matrices given by (2.2.1) and (2.2.2) is

$$
\begin{gathered}
\operatorname{Min}-\operatorname{Max}\left(A_{3 \times 3}, B_{3 \times 3}\right)=C_{3 \times 3} \\
=\left[\begin{array}{lll}
0.5 & 0.5 & 0.2 \\
0.3 & 0.2 & 0.2 \\
0.3 & 0.3 & 0.3
\end{array}\right]
\end{gathered}
$$

where, $\quad a_{11}=$ $\min \{\max (0.7,0.2), \max (0.5,0.5), \max (0.2,0.8)\}=$ $\max \{0.7,0.5,0.8\}=0.5$ 
$a_{12}$

$=\min \{\max (0.7,0.3), \max (0.5,0.2), \max (0.2,0.7)\}$

$$
\begin{gathered}
H_{e}(A) \\
=\frac{1}{n \sqrt{e}-1} \sum_{i=1}^{n}\left(\mu_{A}\left(x_{i}\right) e^{1-\mu_{A}\left(x_{i}\right)}\right. \\
\left.+\left(1-\mu_{A}\left(x_{i}\right)\right) e^{\mu_{A}\left(x_{i}\right)}-1\right)
\end{gathered}
$$

Bhandari and Pal (1993) described the idea of fuzzy directed divergence considering the elementary properties of Kullback and Leibler (1951) directed divergence measure. Fuzzy divergence measure as developed by Bhandari and Pal, gives a fuzzy information measure for distinction of a two fuzzy set.

Bhandari and Pal (1993) defined the fuzzy information for discrimination in favour of fuzzy set $A$ against $B$ by

$$
\begin{aligned}
& =\sum_{i=1}^{n}\left[\mu_{A}\left(x_{i}\right) \log \frac{\mu_{A}\left(x_{i}\right)}{\mu_{B}\left(x_{i}\right)}\right. \\
& \left.+\left(1-\mu_{A}\left(x_{i}\right)\right) \log \frac{1-\mu_{A}\left(x_{i}\right)}{1-\mu_{B}\left(x_{i}\right)}\right]
\end{aligned}
$$

Bhandari and Pal (1993) provide the fuzzy entropy by using $I\left(A, A_{F}\right)$ as follows:

$$
H_{D T}(A)=1-\frac{1}{n \ln 2} I\left(A, A_{F}\right)
$$

Bhandari and Pal (1993) defined a divergence measure between two fuzzy sets $\mathrm{R} \& S$ as follows:

$$
\begin{aligned}
& D(R, S)=I(R, S)+I(S, R) \\
& \quad=\sum_{i=1}^{n}\left[\left(\mu_{R}\left(x_{i}\right)-\mu_{S}\left(x_{i}\right)\right) \log \frac{\mu_{R}\left(x_{i}\right)}{\mu_{S}\left(x_{i}\right)}\right. \\
& \left.+\left(\mu_{S}\left(x_{i}\right)-\mu_{R}\left(x_{i}\right)\right) \log \frac{1-\mu_{R}\left(x_{i}\right)}{1-\mu_{S}\left(x_{i}\right)}\right]
\end{aligned}
$$

Definition: Shang and Jiang (1997) pointed out that the measure in Equation (3.7) has a drawback, i.e., when $\mu_{S}\left(x_{i}\right)$ approaches 0 or 1 for any value of $x_{i}$, its value tends toward infinity. Therefore, using the idea of Lin (1991) divergence measure as in equation (3.4), for $R, S \in F S(X)$, Shang and Jiang extended a fuzzy divergence measure (3.7), as: 


$$
D_{S J}(R, S) \sum_{i=1}^{n}\left[\begin{array}{l}
\left.\left(\mu_{R}\left(x_{i}\right)-\mu_{S}\left(x_{i}\right)\right) \ln \frac{\mu_{R}\left(x_{i}\right)}{\left(\frac{\mu_{R}\left(x_{i}\right)+\mu_{S}\left(x_{i}\right)}{2}\right)}\right) \\
\left.+\left(\mu_{S}\left(x_{i}\right)-\mu_{R}\left(x_{i}\right)\right) \ln \frac{1-\mu_{R}\left(x_{i}\right)}{\left(\frac{2-\mu_{R}\left(x_{i}\right)-\mu_{S}\left(x_{i}\right)}{2}\right)}\right)
\end{array}\right]
$$

Some other measure of fuzzy directed divergence measures have been introduced and studied by Pal and Bezdek (1994), Kapur (1997), Fan and Xie (1999), Hooda (2004), Bajaj and Hooda (2010), Kumar et al. (2011), Bhatia and Singh (2013), Tomar

\section{LOGARITHMIC DIVERGENCE MEASURE FOR FUZZY MATRIX}

In this section, a divergence measure for fuzzy matrices $R \& S$ is proposed which is of logarithmic form. The validity of the proposed divergence measure is verified.

Here we define a fuzzy divergence measure for fuzzy matrices:

Definition (2020): Let $F_{M}$ be the set of all fuzzy matrices having $m$ rows and $n$ columns and $X \& Y \in F_{M}$. Then a mapping $J: F_{M} \times F_{M} \rightarrow A$ is called nonprobabilistic divergence measure of fuzzy matrices if and only if

$$
\begin{gathered}
J(X: Y)=\sum_{i=1}^{m} \sum_{j=1}^{n}\left\{\left(x_{i j}-y_{i j}\right) \log \frac{x_{i j}}{y_{i j}}\right\}+\left\{\left(\left(1-x_{i j}\right)-\left(1-y_{i j}\right)\right) \log \frac{1-x_{i j}}{1-y_{i j}}\right\} \\
=\sum_{i=1}^{m} \sum_{j=1}^{n}\left[\left\{\left(x_{i j}-y_{i j}\right) \log \frac{x_{i j}}{y_{i j}}\right\}+\left\{\left(y_{i j}-x_{i j}\right) \log \frac{1-x_{i j}}{1-y_{i j}}\right\}\right]
\end{gathered}
$$

where $x_{i j} \in X \& y_{i j} \in Y$

To show that the proposed measure is a valid measure since it satisfies all the above four axioms which are proving by following theorem:

Theorem 4.1: $J(X: Y) \geq 0$ if $X$ and $Y \in$ $\left[F_{M}\right]_{m \times n}$. and Ohlan (2014), Verma and Sharma (2014), Gupta and Santosh (2014), Ohlan (2015) and etc. These fuzzy divergence measures have application in various fields including decision making problems, fuzzy clustering, artificial intelligence etc.

a. $\quad J(X: Y) \geq 0$

b. $\quad J(X: Y)=0$ when $X \& Y$ are equal fuzzy matrix

c. $J(X: Y)=J(Y: X)$ i.e. divergence measure is symmetric in nature.

d. $\quad J(X: Y)$ is convex in $X$ and $Y$.

Thus we say that a measure is information theoretic divergence measure for fuzzy matrices if it satisfies axioms (a) to (d).

Here we proposed a divergence measure for fuzzy matrices $X$ and $Y$ of order $m \times n$ which is logarithmic in nature as follows:

Proof: It is trivial that the measure is nonnegative for each $a \& b$ (where $a=x_{i j} \in$ $\left.X \& b=y_{i j} \in Y\right)$.

Theorem 4.2: $J(X: Y)=0$ if $X=Y$ or $x_{i j}=y_{i j}$.

Proof: We have 


$$
J(X: Y)=\sum_{i=1}^{m} \sum_{j=1}^{n}\left[\left\{\left(x_{i j}-y_{i j}\right) \log \frac{x_{i j}}{y_{i j}}\right\}+\left\{\left(y_{i j}-x_{i j}\right) \log \frac{1-x_{i j}}{1-y_{i j}}\right\}\right]
$$

And if $X=Y$ or $x_{i j}=y_{i j}$ then

Hence proved.

$$
\begin{gathered}
J(X: Y)=\sum_{i=1}^{m} \sum_{j=1}^{n}\left[\left(x_{i j}-x_{i j}\right) \log \frac{x_{i j}}{x_{i j}}+\left(x_{i j}-x_{i j}\right) \log \frac{1-x_{i j}}{1-x_{i j}}\right] \\
J(X: Y)=\sum_{i=1}^{m} \sum_{j=1}^{n}[0+0]=\sum_{i=1}^{m} \sum_{j=1}^{n}[0] \\
J(X: Y)=0
\end{gathered}
$$

Theorem 4.3: $J(X: Y)=J(Y: X)$.

Proof: To prove we show $J(X: Y)-J(Y: X)=0$

$$
\begin{aligned}
& J(X: Y)=\sum_{i=1}^{m} \sum_{j=1}^{n}\left[\left\{\left(x_{i j}-y_{i j}\right) \log \frac{x_{i j}}{y_{i j}}\right\}+\left\{\left(y_{i j}-x_{i j}\right) \log \frac{1-x_{i j}}{1-y_{i j}}\right\}\right] \\
& J(Y: X)=\sum_{i=1}^{m} \sum_{j=1}^{n}\left[\left\{\left(y_{i j}-x_{i j}\right) \log \frac{y_{i j}}{x_{i j}}\right\}+\left\{\left(x_{i j}-y_{i j}\right) \log \frac{1-y_{i j}}{1-x_{i j}}\right\}\right] \\
& J(X: Y)-J(Y: X)=\sum_{i=1}^{m} \sum_{j=1}^{n}\left[\begin{array}{l}
\left(x_{i j}-y_{i j}\right)\left(\log \frac{x_{i j}}{y_{i j}}+\log \frac{1-y_{i j}}{1-x_{i j}}\right) \\
+\left(y_{i j}-x_{i j}\right)\left(\log \frac{y_{i j}}{x_{i j}}+\log \frac{1-x_{i j}}{1-y_{i j}}\right)
\end{array}\right] \\
& =\sum_{i=1}^{m} \sum_{j=1}^{n}\left[\begin{array}{c}
\left(x_{i j}-y_{i j}\right)\left(\log \frac{x_{i j}}{y_{i j}}+\log \frac{1-y_{i j}}{1-x_{i j}}\right) \\
-\left(x_{i j}-y_{i j}\right)\left(\log \frac{y_{i j}}{x_{i j}}+\log \frac{1-x_{i j}}{1-y_{i j}}\right)
\end{array}\right] \\
& =\sum_{i=1}^{m} \sum_{j=1}^{n}\left[\left(x_{i j}-y_{i j}\right)\left(\left(\log \frac{x_{i j}}{y_{i j}}+\log \frac{1-y_{i j}}{1-x_{i j}}\right)-\left(\log \frac{y_{i j}}{x_{i j}}+\log \frac{1-x_{i j}}{1-y_{i j}}\right)\right)\right] \\
& =\sum_{i=1}^{m} \sum_{j=1}^{n}\left[\left(x_{i j}-y_{i j}\right)\left(\left(\log \frac{x_{i j}}{y_{i j}} \frac{1-y_{i j}}{1-x_{i j}}\right)-\left(\log \frac{y_{i j}}{x_{i j}} \frac{1-x_{i j}}{1-y_{i j}}\right)\right)\right] \\
& =\sum_{i=1}^{m} \sum_{j=1}^{n}\left[\left(x_{i j}-y_{i j}\right)\left(\log \frac{x_{i j}}{y_{i j}} \frac{1-y_{i j}}{1-x_{i j}} \frac{y_{i j}}{x_{i j}} \frac{1-x_{i j}}{1-y_{i j}}\right)\right] \\
& =\sum_{i=1}^{m} \sum_{j=1}^{n}\left[\left(x_{i j}-y_{i j}\right) \log (1)\right] \\
& J(X: Y)-J(Y: X)=\sum_{i=1}^{m} \sum_{j=1}^{n}\left[\left(x_{i j}-y_{i j}\right)(0)\right]=\sum_{i=1}^{m} \sum_{j=1}^{n}[0] \\
& J(X: Y)-J(Y: X)=0
\end{aligned}
$$

Theorem 4.4: $J(X: Y)$ is convex in $X$ and $Y$. 
Proof: First we prove $J(X: Y)$ convex in $X$.

$$
\begin{gathered}
J(X: Y)=\sum_{i=1}^{m} \sum_{j=1}^{n}\left[\left\{\left(x_{i j}-y_{i j}\right) \log \frac{x_{i j}}{y_{i j}}\right\}+\left\{\left(y_{i j}-x_{i j}\right) \log \frac{1-x_{i j}}{1-y_{i j}}\right\}\right] \\
\frac{\partial J(X: Y)}{\partial x_{i j}}=\sum_{i=1}^{m} \sum_{j=1}^{n}\left[\left(x_{i j}-y_{i j}\right)\left(\frac{1}{x_{i j}}\right)+\log \frac{x_{i j}}{y_{i j}}+\left(y_{i j}-x_{i j}\right)\left(\frac{-1}{1-x_{i j}}\right)+\log \frac{1-x_{i j}}{1-y_{i j}}(-1)\right]
\end{gathered}
$$

When $X=Y$ or $x_{i j}=y_{i j}$, then

$$
\begin{gathered}
\frac{\partial J(X: Y)}{\partial x_{i j}}=\sum_{i=1}^{m} \sum_{j=1}^{n}\left[\left(x_{i j}-x_{i j}\right)\left(\frac{1}{x_{i j}}\right)+\log \frac{x_{i j}}{x_{i j}}+\left(x_{i j}-x_{i j}\right)\left(\frac{-1}{1-x_{i j}}\right)+\log \frac{1-x_{i j}}{1-x_{i j}}(-1)\right] \\
\frac{\partial J(X: Y)}{\partial x_{i j}}=\sum_{i=1}^{m} \sum_{j=1}^{n}[0+\log (1)+0-\log (1)]=0 \\
\frac{\partial^{2} J(X: Y)}{\partial x_{i j}^{2}}=\sum_{i=1}^{m} \sum_{j=1}^{n}\left[\left(x_{i j}-y_{i j}\right)\left(\frac{-1}{x_{i j}^{2}}\right)+\left(\frac{1}{x_{i j}}\right)+\left(\frac{1}{x_{i j}}\right)+\left(y_{i j}-x_{i j}\right)\left(\frac{-1}{\left(1-x_{i j}\right)^{2}}\right)\right. \\
\left.+\left(\frac{1}{1-x_{i j}}\right)+\left(\frac{1}{1-x_{i j}}\right)\right] \\
\frac{\partial^{2} J(X: Y)}{\partial x_{i j}^{2}}=\sum_{i=1}^{m} \sum_{j=1}^{n}\left[\left(x_{i j}-y_{i j}\right)\left(\frac{-1}{x_{i j}^{2}}\right)+\left(\frac{2}{x_{i j}}\right)+\left(y_{i j}-x_{i j}\right)\left(\frac{-1}{\left(1-x_{i j}\right)^{2}}\right)+\left(\frac{2}{1-x_{i j}}\right)\right]
\end{gathered}
$$

When $X=Y$ or $x_{i j}=y_{i j}$, then

$$
\begin{gathered}
\frac{\partial^{2} J(X: Y)}{\partial x_{i j}{ }^{2}}=\sum_{i=1}^{m} \sum_{j=1}^{n}\left[\left(x_{i j}-x_{i j}\right)\left(\frac{-1}{x_{i j}^{2}}\right)+\left(\frac{2}{x_{i j}}\right)+\left(x_{i j}-x_{i j}\right)\left(\frac{-1}{\left(1-x_{i j}\right)^{2}}\right)+\left(\frac{2}{1-x_{i j}}\right)\right] \\
\frac{\partial^{2} J(X: Y)}{\partial x_{i j}{ }^{2}}=\sum_{i=1}^{m} \sum_{j=1}^{n}\left[0+\left(\frac{2}{x_{i j}}\right)+0+\left(\frac{2}{1-x_{i j}}\right)\right]=\sum_{i=1}^{m} \sum_{j=1}^{n}\left[\left(\frac{2}{x_{i j}}\right)+\left(\frac{2}{1-x_{i j}}\right)\right] \\
\frac{\partial^{2} J(X: Y)}{\partial x_{i j}{ }^{2}}=\sum_{i=1}^{m} \sum_{j=1}^{n}\left[\frac{2\left(1-x_{i j}+x_{i j}\right)}{x_{i j}\left(1-x_{i j}\right)}\right]=\sum_{i=1}^{m} \sum_{j=1}^{n}\left[\frac{2(1)}{x_{i j}\left(1-x_{i j}\right)}\right]>0 \\
\frac{\partial^{2} J(X: Y)}{\partial x_{i j}{ }^{2}}=\sum_{i=1}^{m} \sum_{j=1}^{n}\left[\frac{2(1)}{x_{i j}\left(1-x_{i j}\right)}\right]>0 \text { where } x_{i j}>0
\end{gathered}
$$

The proof of the theorem shows $J(X: Y)$ is a convex function of $X$.

Similarly, we can show that $J(X: Y)$ is a convex function of $Y$.

The proposed divergence measure is a valid divergence measure of fuzzy matrices, satisfies all the four axioms.
a. $J(X: Y)=J\left(X^{c}: Y^{c}\right)$
b. $J[(X \cup Y):(X \cap Y)]=J(X: Y)$
c. $J(X:(X \cup Y))=J(Y:(X \cap Y))$

\section{Properties of FuZZY DiVERGENCE} MEASURE

In this section, some properties of proposed measure are proved in the form of theorems. Theorem 5.1: Let $X$ and $Y \in\left[F_{M}\right]_{m \times n}$, then the following properties are satisfied by $J(X: Y)$. 
d. $J(X:(X \cap Y))=J(Y:(X \cup Y)$

Proof: To prove these theorems, we define both fuzzy matrices into two sets as given below:
$S_{1}=\left\{x_{i j}\right.$ or $y_{i j} ; x_{i j} \in X$ or $\left.\left.y_{i j} \in Y ; x_{i j} \geq y_{i j}\right\}\right\}$ $S_{2}=\left\{x_{i j}\right.$ or $y_{i j} ; x_{i j} \in X$ or $\left.\left.y_{i j} \in Y ; x_{i j}<y_{i j}\right\}\right\}$

(a) We have

$J\left(X^{c}: Y^{c}\right)$

$$
J(X: Y)=\sum_{i=1}^{m} \sum_{j=1}^{n}\left[\left\{\left(x_{i j}-y_{i j}\right) \log \frac{x_{i j}}{y_{i j}}\right\}+\left\{\left(y_{i j}-x_{i j}\right) \log \frac{1-x_{i j}}{1-y_{i j}}\right\}\right]
$$

$$
\begin{gathered}
=\sum_{i=1}^{m} \sum_{j=1}^{n}\left[\left(\left(1-x_{i j}\right)-\left(1-y_{i j}\right)\right) \log \frac{1-x_{i j}}{1-y_{i j}}+\left\{\left(\left(1-y_{i j}\right)-\left(1-x_{i j}\right)\right) \log \frac{1-\left(1-x_{i j}\right)}{1-\left(1-y_{i j}\right)}\right\}\right] \\
J\left(X^{c}: Y^{c}\right) \sum_{i=1}^{m} \sum_{j=1}^{n}\left[\left(y_{i j}-x_{i j}\right) \log \frac{1-x_{i j}}{1-y_{i j}}+\left(x_{i j}-y_{i j}\right) \log \frac{x_{i j}}{y_{i j}}\right]
\end{gathered}
$$

$J\left(X^{c}: Y^{c}\right)=J(X: Y)$

(b) We have

$J[(X \cup Y):(X \cap Y)]$

$$
\begin{aligned}
& =\sum_{i=1}^{m} \sum_{j=1}^{n}\left[\begin{array}{c}
\left(\max \left(x_{i j}, y_{i j}\right)-\min \left(x_{i j}, y_{i j}\right) \log \frac{\max \left(x_{i j}, y_{i j}\right)}{\min \left(x_{i j}, y_{i j}\right)}\right) \\
+\left(\min \left(x_{i j}, y_{i j}\right)-\max \left(x_{i j}, y_{i j}\right)\right) \log \frac{1-\max \left(x_{i j}, y_{i j}\right)}{1-\min \left(x_{i j}, y_{i j}\right)}
\end{array}\right] \\
& =\sum_{x_{i j}, y_{i j} \in S_{1}}\left[\left\{\left(x_{i j}-y_{i j}\right) \log \frac{x_{i j}}{y_{i j}}\right\}+\left\{\left(y_{i j}-x_{i j}\right) \log \frac{1-x_{i j}}{1-y_{i j}}\right\}\right] \\
& +\sum_{x_{i j}, y_{i j} \in S_{2}}\left[\left\{\left(x_{i j}-y_{i j}\right) \log \frac{x_{i j}}{y_{i j}}\right\}+\left\{\left(y_{i j}-x_{i j}\right) \log \frac{1-x_{i j}}{1-y_{i j}}\right\}\right] \\
& =\left\{\sum_{x_{i j}, y_{i j} \in S_{1}}\left[\left\{\left(x_{i j}-y_{i j}\right) \log \frac{x_{i j}}{y_{i j}}\right\}\right]+\sum_{x_{i j}, y_{i j} \in S_{2}}\left[\left\{\left(x_{i j}-y_{i j}\right) \log \frac{x_{i j}}{y_{i j}}\right\}\right]\right\} \\
& +\left\{\sum_{x_{i j}, y_{i j} \in S_{1}}\left[\left(y_{i j}-x_{i j}\right) \log \frac{1-x_{i j}}{1-y_{i j}}\right]+\sum_{x_{i j}, y_{i j} \in S_{2}}\left[\left(y_{i j}-x_{i j}\right) \log \frac{1-x_{i j}}{1-y_{i j}}\right]\right\} \\
& =\sum_{i=1}^{m} \sum_{j=1}^{n}\left[\left(x_{i j}-y_{i j}\right) \log \frac{x_{i j}}{y_{i j}}\right]+\sum_{i=1}^{m} \sum_{j=1}^{n}\left[\left(y_{i j}-x_{i j}\right) \log \frac{1-x_{i j}}{1-y_{i j}}\right] \\
& =\sum_{i=1}^{m} \sum_{j=1}^{n}\left[\left\{\left(x_{i j}-y_{i j}\right) \log \frac{x_{i j}}{y_{i j}}\right\}+\left\{\left(y_{i j}-x_{i j}\right) \log \frac{1-x_{i j}}{1-y_{i j}}\right\}\right]=J(X: Y)
\end{aligned}
$$

Hence proved.

Taking left hand side, we have

(c) $J(X:(X \cup Y))=J(Y:(X \cap Y))$ 
Taking R.H.S,

$$
\begin{aligned}
& J(X:(X \cup Y))=\sum_{i=1}^{m} \sum_{j=1}^{n}\left[\begin{array}{c}
\left(x_{i j}-\max \left(x_{i j}, y_{i j}\right)\right) \log \frac{x_{i j}}{\max \left(x_{i j}, y_{i j}\right)} \\
\left.+\left(\max \left(x_{i j}, y_{i j}\right)\right)-x_{i j}\right) \log \frac{1-x_{i j}}{1-\max \left(x_{i j}, y_{i j}\right)}
\end{array}\right] \\
& =\sum_{x_{i j}, y_{i j} \in S_{1}}\left[\left(x_{i j}-x_{i j}\right) \log \frac{x_{i j}}{x_{i j}}+\left(x_{i j}-x_{i j}\right) \log \frac{1-x_{i j}}{1-x_{i j}}\right] \\
& +\sum_{x_{i j}, y_{i j} \in S_{2}}\left[\left\{\left(x_{i j}-y_{i j}\right) \log \frac{x_{i j}}{y_{i j}}\right\}+\left\{\left(y_{i j}-x_{i j}\right) \log \frac{1-x_{i j}}{1-y_{i j}}\right\}\right] \\
& =\sum_{x_{i j}, y_{i j} \in S_{2}}\left[\left\{\left(x_{i j}-y_{i j}\right) \log \frac{x_{i j}}{y_{i j}}\right\}+\left\{\left(y_{i j}-x_{i j}\right) \log \frac{1-x_{i j}}{1-y_{i j}}\right\}\right]
\end{aligned}
$$

$$
\begin{gathered}
J(Y:(X \cap Y))=\sum_{i=1}^{m} \sum_{j=1}^{n}\left[\left\{\left(\min \left(x_{i j}, y_{i j}\right)-y_{i j}\right) \log \frac{\min \left(x_{i j}, y_{i j}\right)}{y_{i j}}\right\}\right. \\
\left.+\left\{\left(y_{i j}-\min \left(x_{i j}, y_{i j}\right)\right) \log \frac{1-\min \left(x_{i j}, y_{i j}\right)}{1-y_{i j}}\right\}\right] \\
=\sum_{x_{i j}, y_{i j} \in S_{1}}\left[\left(y_{i j}-y_{i j}\right) \log \frac{y_{i j}}{y_{i j}}+\left(y_{i j}-y_{i j}\right) \log \frac{1-y_{i j}}{1-y_{i j}}\right] \\
+\sum_{x_{i j}, y_{i j} \in S_{2}}\left[\left\{\left(x_{i j}-y_{i j}\right) \log \frac{x_{i j}}{y_{i j}}\right\}+\left\{\left(y_{i j}-x_{i j}\right) \log \frac{1-x_{i j}}{1-y_{i j}}\right)\right],=\sum_{x_{i j}, y_{i j} \in S_{2}}\left[\left\{\left(x_{i j}-y_{i j}\right) \log \frac{x_{i j}}{y_{i j}}\right\}+\right. \\
\left.\left\{\left(y_{i j}-x_{i j}\right) \log \frac{1-x_{i j}}{1-y_{i j}}\right\}\right]
\end{gathered}
$$

\section{L.H.S = R.H.S.}

(d) $J(X:(X \cap Y))=J(Y:(X \cup Y))$

Evaluating L.H.S. ,

Taking R.H.S. ,

$$
\begin{aligned}
& J(X:(X \cap Y))=\sum_{i=1}^{m} \sum_{j=1}^{n}\left[\begin{array}{c}
\left(x_{i j}-\min \left(x_{i j}, y_{i j}\right)\right) \log \frac{x_{i j}}{\min \left(x_{i j}, y_{i j}\right)} \\
+\left(\min \left(x_{i j}, y_{i j}\right)-x_{i j}\right) \log \frac{1-x_{i j}}{1-\min \left(x_{i j}, y_{i j}\right)}
\end{array}\right] \\
& =\sum_{x_{i j}, y_{i j} \in S_{1}}\left[\left\{\left(x_{i j}-y_{i j}\right) \log \frac{x_{i j}}{y_{i j}}\right\}+\left\{\left(y_{i j}-x_{i j}\right) \log \frac{1-x_{i j}}{1-y_{i j}}\right\}\right] \\
& +\sum_{x_{i j}, y_{i j} \in S_{2}}\left[\left(x_{i j}-x_{i j}\right) \log \frac{x_{i j}}{x_{i j}}+\left(x_{i j}-x_{i j}\right) \log \frac{1-x_{i j}}{1-x_{i j}}\right] \\
& =\sum_{x_{i j}, y_{i j} \in S_{1}}\left[\left\{\left(x_{i j}-y_{i j}\right) \log \frac{x_{i j}}{y_{i j}}\right\}+\left\{\left(y_{i j}-x_{i j}\right) \log \frac{1-x_{i j}}{1-y_{i j}}\right\}\right]
\end{aligned}
$$




$$
\begin{aligned}
& J(Y:(X \cup Y))=\sum_{i=1}^{m} \sum_{j=1}^{n}\left[\begin{array}{c}
\left(\max \left(x_{i j}, y_{i j}\right)-y_{i j}\right) \log \frac{\max \left(x_{i j}, y_{i j}\right)}{y_{i j}} \\
+\left(y_{i j}-\max \left(x_{i j}, y_{i j}\right)\right) \log \frac{1-\max \left(x_{i j}, y_{i j}\right)}{1-y_{i j}}
\end{array}\right] \\
& =\sum_{x_{i j}, y_{i j} \in S_{1}}\left[\left\{\left(x_{i j}-y_{i j}\right) \log \frac{x_{i j}}{y_{i j}}\right\}+\left\{\left(y_{i j}-x_{i j}\right) \log \frac{1-x_{i j}}{1-y_{i j}}\right\}\right] \\
& +\sum_{x_{i j}, y_{i j} \in S_{2}}\left[\left(x_{i j}-x_{i j}\right) \log \frac{x_{i j}}{x_{i j}}+\left(x_{i j}-x_{i j}\right) \log \frac{1-x_{i j}}{1-x_{i j}}\right] \\
& =\sum_{x_{i j}, y_{i j} \in S_{1}}\left[\left\{\left(x_{i j}-y_{i j}\right) \log \frac{x_{i j}}{y_{i j}}\right\}+\left\{\left(y_{i j}-x_{i j}\right) \log \frac{1-x_{i j}}{1-y_{i j}}\right\}\right]
\end{aligned}
$$

Thus L.H.S = R.H.S.

Corollary 5.1: If $X$ and $Y \in\left[F_{M}\right]_{m \times n}$ then we have $J(X:(X \cup Y))+J(X:(X \cap Y))=J(X: Y)$
Proof: From the part $\mathrm{c}$ and $\mathrm{d}$ of theorem (5.1), we have As

And also,

$$
J(X:(X \cup Y))=\sum_{x_{i j}, y_{i j} \in S_{2}}\left[\left\{\left(x_{i j}-y_{i j}\right) \log \frac{x_{i j}}{y_{i j}}\right\}+\left\{\left(y_{i j}-x_{i j}\right) \log \frac{1-x_{i j}}{1-y_{i j}}\right\}\right]
$$

And now,

$$
J(X:(X \cap Y))=\sum_{x_{i j}, y_{i j} \in S_{1}}\left[\left\{\left(x_{i j}-y_{i j}\right) \log \frac{x_{i j}}{y_{i j}}\right\}+\left\{\left(y_{i j}-x_{i j}\right) \log \frac{1-x_{i j}}{1-y_{i j}}\right\}\right]
$$

$$
\begin{aligned}
& J(X:(X \cup Y))+J(X:(X \cap Y))=\left[\begin{array}{l}
\sum_{x_{i j}, y_{i j} \in S_{2}}\left[\left\{\left(x_{i j}-y_{i j}\right) \log \frac{x_{i j}}{y_{i j}}\right\}+\left\{\left(y_{i j}-x_{i j}\right) \log \frac{1-x_{i j}}{1-y_{i j}}\right\}\right] \\
\left.+\sum_{x_{i j}, y_{i j} \in S_{1}}\left[\left\{\left(x_{i j}-y_{i j}\right) \log \frac{x_{i j}}{y_{i j}}\right\}+\left\{\left(y_{i j}-x_{i j}\right) \log \frac{1-x_{i j}}{1-y_{i j}}\right\}\right]\right]
\end{array}\right. \\
& J(X:(X \cup Y))+J(X:(X \cap Y))=\sum_{i=1}^{m} \sum_{j=1}^{n}\left[\left\{\left(x_{i j}-y_{i j}\right) \log \frac{x_{i j}}{y_{i j}}\right\}+\left\{\left(y_{i j}-x_{i j}\right) \log \frac{1-x_{i j}}{1-y_{i j}}\right\}\right] \\
& =J(X: Y)
\end{aligned}
$$

Hence Proved.

Proof: Again from the part $\mathrm{c}$ and $\mathrm{d}$ of Corollary 5.2: If $X$ and $Y \in\left[F_{M}\right]_{m \times n}$ then we have $J(Y:(X \cup Y))+J(Y:(X \cap Y))=J(X: Y)$ theorem (5.1) we can prove it easily.

As

$$
J(Y:(X \cap Y))=\sum_{x_{i j}, y_{i j} \in S_{2}}\left[\left\{\left(x_{i j}-y_{i j}\right) \log \frac{x_{i j}}{y_{i j}}\right\}+\left\{\left(y_{i j}-x_{i j}\right) \log \frac{1-x_{i j}}{1-y_{i j}}\right\}\right]
$$

And also, 
And now,

$$
J(Y:(X \cup Y))=\sum_{x_{i j}, y_{i j} \in S_{1}}\left[\left\{\left(x_{i j}-y_{i j}\right) \log \frac{x_{i j}}{y_{i j}}\right\}+\left\{\left(y_{i j}-x_{i j}\right) \log \frac{1-x_{i j}}{1-y_{i j}}\right\}\right]
$$

$$
\begin{array}{r}
J(Y:(X \cup Y))+J(Y:(X \cap Y))=\left[\begin{array}{c}
\sum_{x_{i j}, y_{i j} \in S_{2}}\left[\left\{\left(x_{i j}-y_{i j}\right) \log \frac{x_{i j}}{y_{i j}}\right\}+\left\{\left(y_{i j}-x_{i j}\right) \log \frac{1-x_{i j}}{1-y_{i j}}\right\}\right] \\
\left.+\sum_{x_{i j}, y_{i j} \in S_{1}}\left[\left\{\left(x_{i j}-y_{i j}\right) \log \frac{x_{i j}}{y_{i j}}\right\}+\left\{\left(y_{i j}-x_{i j}\right) \log \frac{1-x_{i j}}{1-y_{i j}}\right\}\right]\right]
\end{array}\right. \\
J(Y:(X \cup Y))+J(Y:(X \cap Y))=\sum_{i=1}^{m} \sum_{j=1}^{n}\left[\left\{\left(x_{i j}-y_{i j}\right) \log \frac{x_{i j}}{y_{i j}}\right\}+\left\{\left(y_{i j}-x_{i j}\right) \log \frac{1-x_{i j}}{1-y_{i j}}\right\}\right] \\
J((X \cup Y): X)=J((X \cap Y): Y) \leq J(X: Y)
\end{array}
$$

Corollary 5.3: If $X$ and $Y \in\left[F_{M}\right]_{m \times n}$ then we have

$J((X \cap Y): X)=J((X \cup Y): Y) \leq J(X: Y)$

Proof: From symmetric property of divergence measure

$J(X:(X \cap Y))=J((X \cap Y): X) \& J((X \cup Y): Y)$

$$
=J(Y:(X \cup Y))
$$

Now from part (d) of theorem 5.1 we have,

$J(X:(X \cap Y))=J(Y:(X \cup Y)$

Hence,

$J((X \cap Y): X)=J((X \cup Y): Y)$

Now by using corollary (5.1) we have,

$J(X:(X \cup Y))+J(X:(X \cap Y))=J(X: Y)$

$J((X \cap Y): X)=J(X:(X \cap Y))$

$$
=J(X: Y)-J(X:(X \cup Y))
$$

Thus,

$$
J((X \cap Y): X)=J((X \cup Y): Y) \leq J(X: Y)
$$

Hence proved.

Corollary 5.4: If $X$ and $Y \in\left[F_{M}\right]_{m \times n}$ then we have
a. $J\left(X: Y^{c}\right)=J\left(X^{c}: Y\right)$
b. $J\left(X: X^{c}\right)=0$ when $x_{i j}=0$ or 1 for all $i$ and $j$.
c. $J\left(X: X^{c}\right)=0$ when $x_{i j}=0.5$ for all $i$ and $j$.

Proof: We have

$$
J(X: Y)=\sum_{i=1}^{m} \sum_{j=1}^{n}\left[\left\{\left(x_{i j}-y_{i j}\right) \log \frac{x_{i j}}{y_{i j}}\right\}+\left\{\left(y_{i j}-x_{i j}\right) \log \frac{1-x_{i j}}{1-y_{i j}}\right\}\right]
$$

(a) First taking left hand side

$$
J\left(X: Y^{c}\right)=\sum_{i=1}^{m} \sum_{j=1}^{n}\left[\left(x_{i j}-\left(1-y_{i j}\right)\right) \log \frac{x_{i j}}{1-y_{i j}}+\left(\left(1-y_{i j}\right)-x_{i j}\right) \log \frac{1-x_{i j}}{1-\left(1-y_{i j}\right)}\right]
$$

Proof: By symmetric properties of divergence measure,

$$
\begin{aligned}
J(X:(X \cup Y)) & =J((X \cup Y): X) \& J((X \cap Y): Y) \\
& =J(Y:(X \cap Y))
\end{aligned}
$$

Now by using part (c) of theorem (5.1) we have,

Hence,

$$
J(X:(X \cup Y))=J(Y:(X \cap Y))
$$

$$
J((X \cup Y): X)=J((X \cap Y): Y)
$$

Now using corollary (5.2) we have,

$$
\begin{gathered}
J(Y:(X \cup Y))+J(Y:(X \cap Y))=J(X: Y) \\
J((X \cap Y): Y)=J(Y:(X \cap Y)) \\
=J(X: Y)-J(Y:(X \cup Y)) \\
\text { Therefore, } \\
J((X \cup Y): X)=J((X \cap Y): Y) \leq J(X: Y)
\end{gathered}
$$

Hence Proved.

Theorem 5.2: Let $X$ and $Y \in\left[F_{M}\right]_{m \times n}$ then the following properties are satisfied by $J(X: Y)$. 


$$
J\left(X: Y^{c}\right)=\sum_{i=1}^{m} \sum_{j=1}^{n}\left[\left(x_{i j}+y_{i j}-1\right) \log \frac{x_{i j}}{1-y_{i j}}+\left(1-y_{i j}-x_{i j}\right) \log \frac{1-x_{i j}}{y_{i j}}\right]
$$

Now, taking right hand side

$$
\begin{gathered}
J\left(X^{c}: Y\right)=\sum_{i=1}^{m} \sum_{j=1}^{n}\left[\left(\left(1-x_{i j}\right)-y_{i j}\right) \log \frac{1-x_{i j}}{y_{i j}}+\left(y_{i j}-\left(1-x_{i j}\right)\right) \log \frac{1-\left(1-x_{i j}\right)}{\left(1-y_{i j}\right)}\right] \\
J\left(X^{c}: Y\right)=\sum_{i=1}^{m} \sum_{j=1}^{n}\left[\left(\left(1-x_{i j}-y_{i j}\right)\right) \log \frac{1-x_{i j}}{y_{i j}}+\left(x_{i j}+y_{i j}-1\right) \log \frac{x_{i j}}{\left(1-y_{i j}\right)}\right]
\end{gathered}
$$

\section{L.H.S. = R.H.S.}

(b) We have

$$
\begin{gathered}
J\left(X: X^{c}\right)=\sum_{i=1}^{m} \sum_{j=1}^{n}\left[\left(x_{i j}-\left(1-x_{i j}\right)\right) \log \frac{x_{i j}}{\left(1-x_{i j}\right)}+\left(\left(1-x_{i j}\right)-x_{i j}\right) \log \frac{\left(1-x_{i j}\right)}{1-\left(1-x_{i j}\right)}\right] \\
J\left(X: X^{c}\right)=\sum_{i=1}^{m} \sum_{j=1}^{n}\left[(-1) \log \frac{x_{i j}}{\left(1-x_{i j}\right)}+\left(1-2 x_{i j}\right) \log \frac{1-x_{i j}}{x_{i j}}\right]
\end{gathered}
$$

When $x_{i j}=0$ or 1 or 0.5 for all $i$ and $j$.

$$
J\left(X: X^{c}\right)=\sum_{i=1}^{m} \sum_{j=1}^{n}[0]=0
$$

Hence Proved.

(c) As we know that,

$$
J\left(X: X^{c}\right)=\sum_{i=1}^{m} \sum_{j=1}^{n}\left[(-1) \log \frac{x_{i j}}{\left(1-x_{i j}\right)}+\left(1-2 x_{i j}\right) \log \frac{1-x_{i j}}{x_{i j}}\right]
$$

When $x_{i j}=0.5$ for all $i$ and $j$, then we have

$$
\begin{gathered}
J\left(X: X^{c}\right)=\sum_{i=1}^{m} \sum_{j=1}^{n}\left[(-1) \log \frac{0.5}{(0.5)}+(-1) \log \frac{0.5}{(0.5)}\right] \\
J\left(X: X^{c}\right)=\sum_{i=1}^{m} \sum_{j=1}^{n}[-2 \log (1)] \\
J\left(X: X^{c}\right)=\sum_{i=1}^{m} \sum_{j=1}^{n}[0]=0
\end{gathered}
$$

Hence proved.

Theorem 5.3: Let $X, Y$ and $Z \in\left[F_{M}\right]_{m \times n}$ then

a) $J(X: Z)+J(Y: Z)-J((X \cup Y): Z)=$ $J((X \cap Y): Z)$

b) $J(X: Z)+J(Y: Z)-J((X \cap Y): Z)=$ $J((X \cup Y): Z)$
Proof: To prove this theorem we define fuzzy matrices into two sets as given below: $S_{1}=\left\{x_{i j}\right.$ or $y_{i j} ; x_{i j} \in X$ or $\left.\left.y_{i j} \in Y ; x_{i j} \geq y_{i j}\right\}\right\}$ $S_{2}=\left\{x_{i j}\right.$ or $y_{i j} ; x_{i j} \in X$ or $\left.\left.y_{i j} \in Y ; x_{i j}<y_{i j}\right\}\right\}$ (a) $\mathrm{We}$ have 


$$
\begin{aligned}
& =J((X \cap Y): Z) \\
& =\left\{\begin{array}{c}
\sum_{i=1}^{m} \sum_{j=1}^{n}\left[\left\{\left(x_{i j}-z_{i j}\right) \log \frac{x_{i j}}{z_{i j}}\right\}+\left\{\left(z_{i j}-x_{i j}\right) \log \frac{1-x_{i j}}{1-z_{i j}}\right\}\right] \\
+\sum_{i=1}^{m} \sum_{j=1}^{n}\left[\left\{\left(y_{i j}-z_{i j}\right) \log \frac{y_{i j}}{z_{i j}}\right\}+\left\{\left(z_{i j}-y_{i j}\right) \log \frac{1-y_{i j}}{1-z_{i j}}\right\}\right] \\
-\sum_{i=1}^{m} \sum_{j=1}^{n}\left[\begin{array}{c}
\left.\left(\max \left(x_{i j}, y_{i j}\right)-z_{i j}\right) \log \frac{\max \left(x_{i j}, y_{i j}\right)}{z_{i j}}\right] \\
\left.+\left(z_{i j}-\max \left(x_{i j}, y_{i j}\right)\right) \log \frac{1-\max \left(x_{i j}, y_{i j}\right)}{1-z_{i j}}\right]
\end{array}\right\}
\end{array}\right.
\end{aligned}
$$

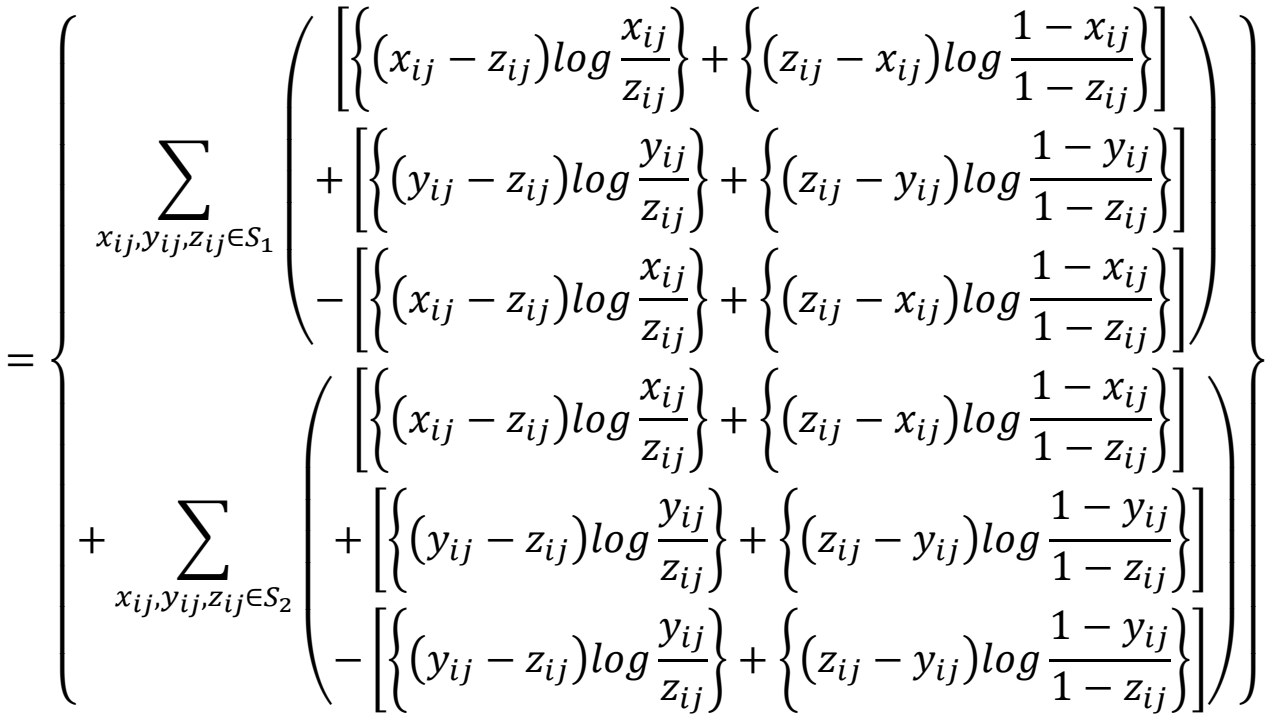

$$
\begin{aligned}
& =\left\{\begin{array}{c}
\sum_{x_{i j}, y_{i j}, z_{i j} \in S_{1}}\left[\left\{\left(y_{i j}-z_{i j}\right) \log \frac{y_{i j}}{z_{i j}}\right\}+\left\{\left(z_{i j}-y_{i j}\right) \log \frac{1-y_{i j}}{1-z_{i j}}\right\}\right] \\
+\sum_{x_{i j}, y_{i j}, z_{i j} \in S_{2}}\left[\left\{\left(x_{i j}-z_{i j}\right) \log \frac{x_{i j}}{z_{i j}}\right\}+\left\{\left(z_{i j}-x_{i j}\right) \log \frac{1-x_{i j}}{1-z_{i j}}\right\}\right]
\end{array}\right\} \\
& =\sum_{i=1}^{m} \sum_{j=1}^{n}\left[\begin{array}{c}
\left(\min \left(x_{i j}, y_{i j}\right)-z_{i j}\right) \log \frac{\min \left(x_{i j}, y_{i j}\right)}{z_{i j}} \\
+\left(z_{i j}-\min \left(x_{i j}, y_{i j}\right)\right) \log \frac{1-\min \left(x_{i j}, y_{i j}\right)}{1-z_{i j}}
\end{array}\right] \\
& J(X: Z)+J(Y: Z)-J((X \cap Y): Z) \\
& =J((X \cup Y): Z)
\end{aligned}
$$

$$
\begin{aligned}
J(X: Z) & +J(Y: Z)-J((X \cup Y): Z) \\
& =J((X \cap Y): Z)
\end{aligned}
$$

Thus we have

a. $J((X \cup Y): Z) \leq J(X: Z)+J(Y: Z)$
Hence Proved.

Corollary 5.5: If $X, Y$ and $Z \in\left[F_{M}\right]_{m \times n}$ then 
b. $\quad J((X \cap Y): Z) \leq J(X: Z)+J(Y: Z)$

\section{Proof:}

(a) Using part 'b' of theorem (5.3) we know that,

$$
\begin{aligned}
J((X \cup Y): Z)= & J(X: Z)+J(Y: Z) \\
& -J((X \cap Y): Z)
\end{aligned}
$$

Since $J((X \cap Y): Z) \geq 0$,

Hence,

Hence Proved.

$$
J((X \cup Y): Z) \leq J(X: Z)+J(Y: Z)
$$

(b) Using part ' $a$ ' of theorem (5.3) we know that,

$$
\begin{aligned}
J((X \cap Y): Z)= & J(X: Z)+J(Y: Z) \\
& -J((X \cup Y): Z)
\end{aligned}
$$

Since $J((X \cup Y): Z) \geq 0$

Hence,

$$
J((X \cap Y): Z) \leq J(X: Z)+J(Y: Z)
$$

Hence Proved.

\section{APPLICATION IN DeCision MAKING AND DATA REDUCTION}

The proposed measure has application in various decision making problems as well as in data reduction. Here a real life example is considered:

The Department of Statistics, Maharshi Dayanand University, Rohtak has a problem which software should be introduced for the students. We have collected data about this problem from teachers and also from outside about the software in the form of fuzzy set $\left\{\dot{\mathrm{S}}_{1}, \dot{\mathrm{S}}_{2}, \ldots \ldots, \dot{\mathrm{S}}_{6}\right\}$ having parameters $\left\{\dot{\mathrm{P}}_{1}, \dot{\mathrm{P}}_{2}, \ldots \ldots, \dot{\mathrm{P}}_{6}\right\}$. The data is collected by $\dot{\mathrm{S}}_{4}=\left\{\left(\dot{\mathrm{p}}_{1}, 0.6\right),\left(\dot{\mathrm{p}}_{2}, 0.6\right),\left(\dot{\mathrm{p}}_{3}, 0.6\right),\left(\dot{\mathrm{p}}_{4}, 0.2\right),\left(\dot{\mathrm{p}}_{5}, 0.5\right),\left(\dot{\mathrm{p}}_{6}, 0.7\right)\right\}$ $\dot{S}_{5}=\left\{\left(\dot{\mathrm{p}}_{1}, 0.3\right),\left(\dot{\mathrm{p}}_{2}, 0.2\right),\left(\dot{\mathrm{p}}_{3}, 0.3\right),\left(\dot{\mathrm{p}}_{4}, 1.0\right),\left(\dot{\mathrm{p}}_{5}, 0.7\right),\left(\dot{\mathrm{p}}_{6}, 0.2\right)\right\}$

$\dot{\mathrm{S}}_{6}=\left\{\left(\dot{\mathrm{p}}_{1}, 0.4\right),\left(\dot{\mathrm{p}}_{2}, 0.7\right),\left(\dot{\mathrm{p}}_{3}, 0.7\right),\left(\dot{\mathrm{p}}_{4}, 0.7\right),\left(\dot{\mathrm{p}}_{5}, 0.7\right),\left(\dot{\mathrm{p}}_{6}, 0.8\right)\right\}$ interview method. There are six software SPSS, C++, R, MATLAB, C language, TORA from which only one is included in the syllabus for students on the basis of selected parameters. We have taken 8 parameters/attributes based on the software chosen. The parameters are

1. Job efficient

2. Latest

3. Availability of tutor

4. Useful in Statistics

5. Cheap

6. Easy to learn

7. Basic

8. Curriculum related

Now, we have chosen only six parameters from all, some biased parameters are removed. They are:

1. Job efficiency

2. Latest

3. Useful in Statistics

4. Cheap

5. Easy to learn

6. Curriculum related

Now for evaluating six softwares, the following six fuzzy sets will be constructed as:

$\dot{\mathrm{S}}_{1}$

$=\left\{\left(\dot{\mathrm{p}}_{1}, 0.9\right),\left(\dot{\mathrm{p}}_{2}, 0.8\right),\left(\dot{\mathrm{p}}_{3}, 0.9\right),\left(\dot{\mathrm{p}}_{4}, 0.1\right),\left(\dot{\mathrm{p}}_{5}, 0.8\right),\left(\dot{\mathrm{p}}_{6}, 1.0\right)\right\}$ $\dot{S}_{2}$

$=\left\{\left(\dot{\mathrm{p}}_{1}, 0.2\right),\left(\dot{\mathrm{p}}_{2}, 0.3\right),\left(\dot{\mathrm{p}}_{3}, 0.4\right),\left(\dot{\mathrm{p}}_{4}, 1.0\right),\left(\dot{\mathrm{p}}_{5}, 0.2\right),\left(\dot{\mathrm{p}}_{6}, 0.1\right)\right\}$ $\dot{\mathrm{S}}_{3}$

$=\left\{\left(\dot{\mathrm{p}}_{1}, 0.9\right),\left(\dot{\mathrm{p}}_{2}, 0.8\right),\left(\dot{\mathrm{p}}_{3}, 0.8\right),\left(\dot{\mathrm{p}}_{4}, 0.3\right),\left(\dot{\mathrm{p}}_{5}, 0.9\right),\left(\dot{\mathrm{p}}_{6}, 0.9\right)\right\}$ 
Here, a table is presented, these six sets which is further converted in a fuzzy matrix ' $\dot{S}^{\prime}$ having order $(6 \times 6)$ where rows represent software and column represents parameters as follows:

\begin{tabular}{|c|l|l|l|l|l|l|}
\hline Software/parameter & \multicolumn{1}{|c|}{$\dot{\mathrm{p}}_{1}$} & $\dot{\mathrm{p}}_{2}$ & $\dot{\mathrm{p}}_{3}$ & \multicolumn{1}{c|}{$\dot{\mathrm{p}}_{4}$} & $\dot{\mathrm{p}}_{5}$ & $\dot{\mathrm{p}}_{6}$ \\
\hline$\dot{\mathrm{S}}_{1}$ & 0.9 & 0.8 & 0.9 & 0.1 & 0.8 & 0.8 \\
\hline$\dot{\mathrm{S}}_{2}$ & 0.2 & 0.3 & 0.4 & 0.9 & 0.2 & 0.2 \\
\hline$\dot{\mathrm{S}}_{3}$ & 0.9 & 0.8 & 0.8 & 0.5 & 0.9 & 0.9 \\
\hline$\dot{\mathrm{S}}_{4}$ & 0.6 & 0.6 & 0.6 & 0.2 & 0.5 & 0.5 \\
\hline$\dot{\mathrm{S}}_{5}$ & 0.3 & 0.3 & 0.3 & 0.9 & 0.7 & 0.7 \\
\hline$\dot{\mathrm{S}}_{6}$ & 0.4 & 0.7 & 0.7 & 0.7 & 0.7 & 0.5 \\
\hline
\end{tabular}

$$
\dot{S}=\left[\begin{array}{cccccc}
0.9 & 0.8 & 0.9 & 0.1 & 0.8 & 0.8 \\
0.2 & 0.3 & 0.4 & 0.9 & 0.2 & 0.2 \\
0.9 & 0.8 & 0.8 & 0.5 & 0.9 & 0.9 \\
0.6 & 0.6 & 0.6 & 0.2 & 0.5 & 0.5 \\
0.3 & 0.3 & 0.3 & 0.9 & 0.7 & 0.7 \\
0.4 & 0.7 & 0.7 & 0.7 & 0.7 & 0.5
\end{array}\right]
$$

Let $\quad T=\quad T=\left[\begin{array}{llllll}0.8 & 0.6 & 0.9 & 0.8 & 0.5 & 0.9\end{array}\right]$ $\left\{\left(\dot{\mathrm{P}}_{1}, 0.8\right),\left(\dot{\mathrm{P}}_{2}, 0.6\right),\left(\dot{\mathrm{P}}_{3}, 0.9\right),\left(\dot{\mathrm{P}}_{4}, 0.8\right),\left(\dot{\mathrm{P}}_{5}, 0.5\right),\left(\dot{\mathrm{P}}_{6}, 0.9\right)\right\}$ be the standard of various parameters for a particular software provided by the head of department.

Then as a fuzzy row matrix $T$ can be represented as:
Now divide the fuzzy matrix $\dot{S}$ into six row matrices as $\left\{\dot{\mathrm{S}}_{1}, \dot{\mathrm{S}}_{2}, \ldots \ldots, \dot{\mathrm{S}}_{6}\right\}$ Now we will find the divergence between each row matrix and matrix $T$ by using proposed divergence measure

$$
\begin{aligned}
& J(X: Y)= \sum_{i=1}^{m} \sum_{j=1}^{n}\left\{\left(x_{i j}-y_{i j}\right) \log \frac{x_{i j}}{y_{i j}}\right\}+\left\{\left(y_{i j}-x_{i j}\right) \log \frac{1-x_{i j}}{1-y_{i j}}\right\} \\
& J\left(\dot{S}_{1}: T\right)=\left\{\begin{array}{l}
{\left[(0.9-0.8) \log \frac{0.9}{0.8}+(0.8-0.9) \log \frac{1-0.9}{1-0.8}\right]} \\
{\left[(0.2-0.6) \log \frac{0.2}{0.6}+(0.2-0.6) \log \frac{1-0.2}{1-0.6}\right]} \\
{\left[(0.9-0.9) \log \frac{0.9}{0.9}+(0.9-0.9) \log \frac{1-0.9}{1-0.9}\right]} \\
{\left[(0.6-0.8) \log \frac{0.6}{0.8}+(0.6-0.8) \log \frac{1-0.6}{1-0.8}\right]} \\
{\left[(0.3-0.5) \log \frac{0.3}{0.5}+(0.3-0.5) \log \frac{1-0.3}{1-0.5}\right]} \\
{\left[(0.4-0.9) \log \frac{0.4}{0.9}+(0.9-0.4) \log \frac{1-0.4}{1-0.9}\right]}
\end{array}\right\}=1.42566
\end{aligned}
$$

We need to find divergence of $T$ with other matrices $\left(\dot{\mathrm{S}}_{2}, \dot{\mathrm{S}}_{3}, \ldots \ldots, \dot{\mathrm{S}}_{6}\right)$ which are given below:

$$
\begin{gathered}
J\left(\dot{\mathrm{S}}_{2}: T\right)=2.75611 \\
J\left(\dot{\mathrm{S}}_{3}: T\right)=\mathbf{0 . 7 1 7 9 4 5 3} \\
J\left(\dot{\mathrm{S}}_{4}: T\right)=1.42281 \\
J\left(\dot{\mathrm{S}}_{5}: T\right)=1.66764, \quad J\left(\dot{\mathrm{S}}_{6}: T\right)=0.9264 .
\end{gathered}
$$


Third row matrix $\left(\dot{\mathrm{S}}_{3}\right)$ has minimum divergence from the other row matrices. The optimum solution of $T$ is $\mathbf{0 . 7 1 7 9 4 5 3}$ which

The choice of preferred software for students according to all teachers of the department has been shown in the following figure (1).The figure clearly shows that is Software $\dot{S}_{3}$. Thus software $\dot{S}_{3}$ will be best in preference of $\dot{S}_{6}, \dot{S}_{4}, \dot{S}_{1}, \dot{S}_{5}, \dot{S}_{2}$.

software $\left(\dot{\mathrm{S}}_{3}\right)$ has minimum divergence than other softwares. Thus R software is the best for the students according to teachers.

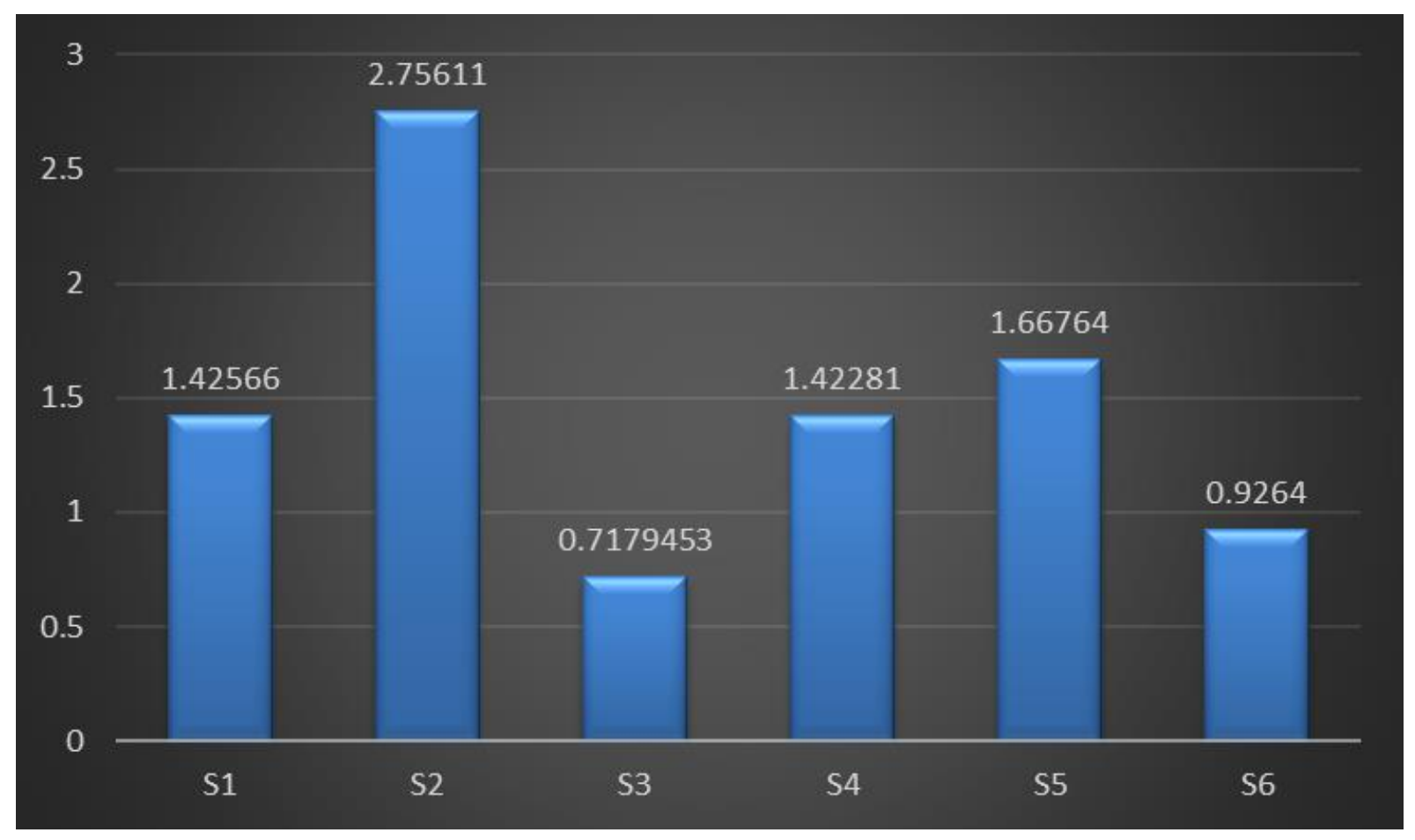

Figure 1: The comparison chart of different software showing divergence

In matrix $\dot{S}$ the columns of matrix are representation the parameters of software, thus for parameter reduction firstly we find the order of significance of these parameters.

Let

$$
Y=
$$

for various software for a particular parameter.

Then as a fuzzy column matrix $Y$ can be represented as:

$$
F=\left[\begin{array}{llllll}
0.8 & 0.2 & 0.8 & 0.4 & 0.3 & 0.5
\end{array}\right]^{T}
$$

Now divide the fuzzy matrix $\dot{S}$ into column matrices as $\left\{F_{1}, F_{2}, \ldots \ldots, F_{6}\right\}$. Now we have to find the divergence between these column $\left\{\left(X_{1}, 0.8\right),\left(X_{2}, 0.2\right),\left(X_{3}, 0.8\right),\left(X_{4}, 0.4\right),\left(X_{5}, 0.3\right),\left(X_{n}\right.\right.$ ant5d $\oint_{\text {s }}$ and matrix $F$ by using proposed be the preferences of head of the department

$$
J(X: Y)=\sum_{i=1}^{m} \sum_{j=1}^{n}\left[\left\{\left(x_{i j}-y_{i j}\right) \log \frac{x_{i j}}{y_{i j}}\right\}+\left\{\left(y_{i j}-x_{i j}\right) \log \frac{1-x_{i j}}{1-y_{i j}}\right\}\right]
$$




$$
J\left(F_{1}: F\right)=\left\{\begin{array}{l}
{\left[(0.9-0.8) \log \frac{0.9}{0.8}+(0.8-0.9) \log \frac{1-0.9}{1-0.8}\right]} \\
{\left[(0.2-0.2) \log \frac{0.2}{0.2}+(0.2-0.2) \log \frac{1-0.2}{1-0.2}\right]} \\
{\left[(0.9-0.8) \log \frac{0.9}{0.8}+(0.8-0.9) \log \frac{1-0.9}{1-0.8}\right]} \\
{\left[(0.6-0.4) \log \frac{0.6}{0.4}+(0.6-0.4) \log \frac{1-0.6}{1-0.4}\right]} \\
{\left[(0.3-0.3) \log \frac{0.3}{0.5}+(0.3-0.3) \log \frac{1-0.3}{1-0.3}\right]} \\
{\left[(0.4-0.5) \log \frac{0.4}{0.5}+(0.5-0.4) \log \frac{1-0.4}{1-0.5}\right]}
\end{array}\right\}=\mathbf{0 . 1 5 8 4 8 2}
$$

Similarly, we may find divergence of $F$ with other matrices $\left(F_{2}, F_{3}, \ldots \ldots, F_{6}\right)$ which are given below:

$$
\begin{array}{r}
J\left(F_{2}: F\right)=\mathbf{0 . 1 6 7 4 4} \\
J\left(F_{3}: F\right)=\mathbf{0 . 2 6 4 4 4 4} \\
J\left(F_{4}: F\right)=\mathbf{3 . 3 1 1 5 6 2} \\
J\left(F_{5}: F\right)=\mathbf{0 . 3 4 7 2 0 9} \\
J\left(F_{6}: F\right)=\mathbf{0 . 5 4 4 8 8 6 .}
\end{array}
$$

The minimum divergence from $Y$ is $\mathbf{0 . 1 5 8 4 8 2}$ which is defined with column matrix $F_{1}$ as compare to other column matrices.

We find the perfect solution of $F$ is $\mathbf{0}$. 158482 which is option $F_{1}$. Thus parameter $P_{1}$ is more significant in comparison to $F_{2}, F_{3}, F_{5}, F_{6}, F_{4}$.

Now we have to take away parameters which do not change the order of preference of software or maintain the optimality of decision. Here $F_{4}$ is least significant so we first abolish $F_{4}$ and check the optimality of the

$$
\begin{array}{r}
J\left(B_{1}: B\right)=\mathbf{0 . 3 3 6 2 5} \\
J\left(B_{2}: F\right)=2.72089, \\
J\left(B_{3}: B\right)=0.53733 \\
J\left(B_{4}: B\right)=0.70034, \\
J\left(B_{5}: B\right)=1.63242, \\
J\left(B_{6}: B\right)=0.90299 .
\end{array}
$$

result.

The decision is not optimal after eviction of $F_{4}$ so we cannot remove $F_{4}$.

When we remove $F_{5}$ then

$$
\begin{aligned}
J\left(B_{1}: B\right)=1.42504, & \\
J\left(B_{2}: B\right) & =2.57549, \\
J\left(B_{3}: B\right) & =\mathbf{0 . 3 3 6 2 5} \\
J\left(B_{4}: B\right)=1.24281, & \\
J\left(B_{5}: B\right) & =1.59404, \\
J\left(B_{6}: B\right) & =0.85281 .
\end{aligned}
$$

We can remove $F_{5}$ because optimality maintain after removal of it.

When we remove $F_{2}$ then

$$
\begin{aligned}
J\left(B_{1}: B\right)=1.34047, & \\
J\left(B_{2}: B\right) & =2.59289, \\
J\left(B_{3}: B\right) & =\mathbf{0 . 6 3 2 7 5} \\
J\left(B_{4}: B\right)=1.42281, & \\
J\left(B_{5}: B\right) & =1.50442, \\
J\left(B_{6}: B\right) & =0.90721 .
\end{aligned}
$$

We can remove $F_{2}$ because optimality maintain after removal of it.

Similarly when we remove $F_{6}, F_{1}, F_{4}$ individualy then optimality of decision is not maintained so we can't remove these parameters individually.

When we remove $F_{2} \& F_{5}$ both then

$$
\begin{aligned}
J\left(B_{1}: B\right)=1.15985, & \\
J\left(B_{2}: B\right) & =2.41227, \\
J\left(B_{3}: B\right) & =\mathbf{0 . 2 5 1 0 5} \\
J\left(B_{4}: B\right)=1.42281, & \\
J\left(B_{5}: B\right) & =1.43082, \\
J\left(B_{6}: B\right) & =0.83362 .
\end{aligned}
$$

Here we can see from the result, solution of the problem has not changed, decision is maintained so we can remove these parameters. 
Since solution is optimal so we can remove any subset from these given parameters set.
Thus we can eliminate the following parameters subset $\left\{\left\{F_{2}, F_{5}\right\},\left\{F_{2}\right\},\left\{F_{5}\right\}\right\}$.

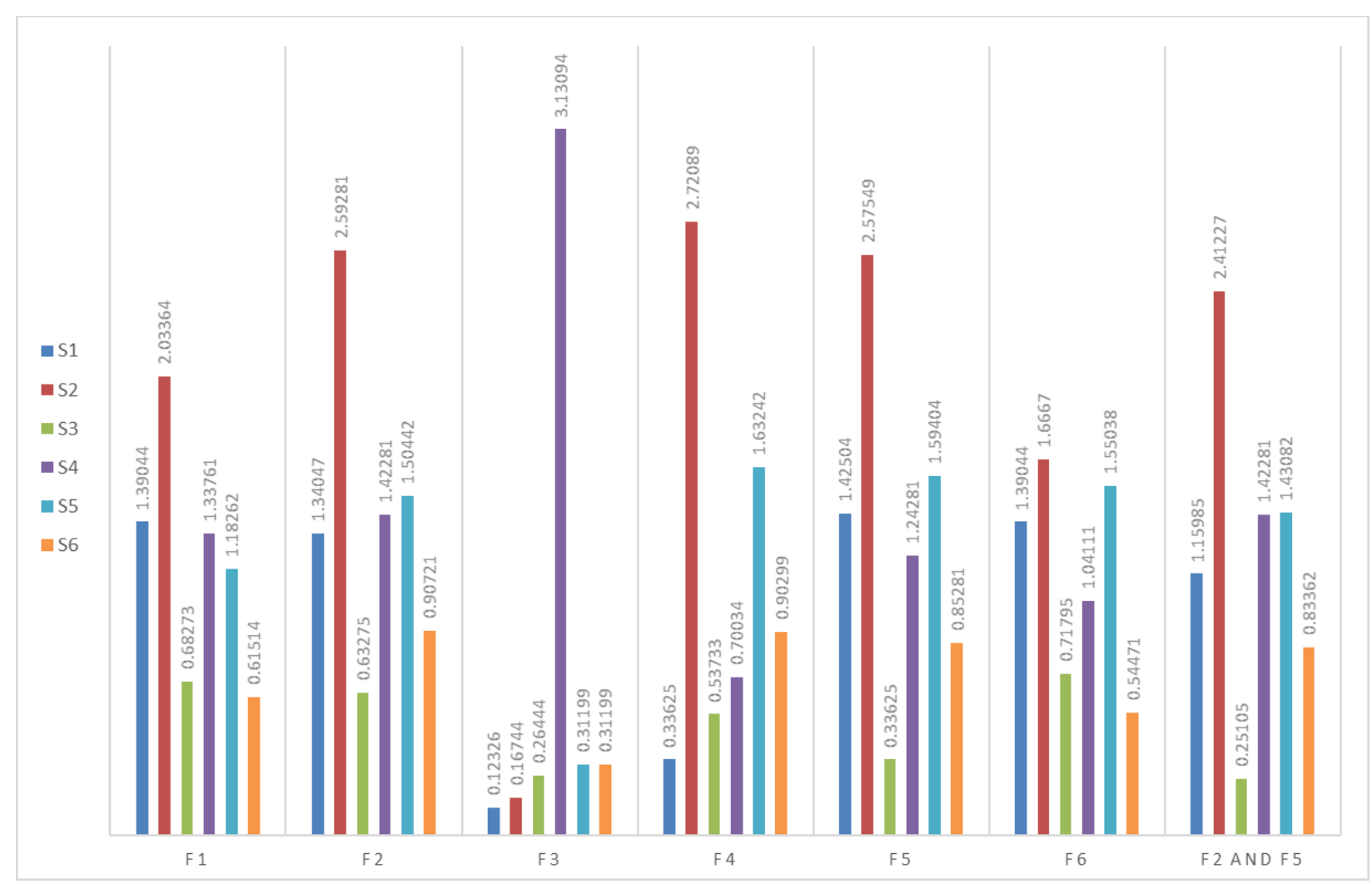

Figure 2: The comparison chart of Feature selection showing decision maintenance

To reduce any feature we check divergence between features and then reduce one by one feature to find out which feature removal does not affect the result. The figure (2) shows only removal of $F_{2}, F_{5}$ and both $F_{2}$ and $F_{5}$ maintain the optimality of the result.

\section{CONCLUSION}

The chapter introduced logarithmic divergence measure for fuzzy matrix. Proposed measure is a valid measure as it satisfies all the axioms. Properties in the form of theorems are also proved of the measure. An application of the measure is presented in decision making problem and parameter reduction problem. We may eclectic the best alternative in preference to other available alternatives in decision making problem. We remove those features which have inconsequential to make decision and after eviction of these features decision is maintained in feature selection problem. Finally, the proposed divergence measure is applied on a case-study to check its real application. 


\section{REFERENCES}

[1]. Bajaj R. K. \& Hooda D. S. (2010), “On Some New Generalized Measures of Fuzzy Information", World Academy of Science, Engineering and Technology, 62, 747-753.

[2].Bajaj, R. K. \& Hooda D. S. (2010), "Generalized Measure of Fuzzy Directed- Divergence: Total Ambiguity and Information Improvement", Journal of Applied Mathematics, Statistics and Informatics, 2, 31-44.

[3].Bhatia P. K. \& Singh S. (2013), "On Some Divergence Measures Between Fuzzy Sets and Aggregation Operations", Advanced Modeling and Optimization, 15(2), 235-248.

[4].Ben M. Bassat (1978), "F-entropies, Probability of Error, and Feature Selection", Inform. Contr., vol. 39, pp. 227-242.

[5].Bhandari D. \& Pal N.R. (1993), "Some New Information Measures for Fuzzy Sets", Information Sciences, 67, 209228.

[6].Chen C. H. (1973), "Statistical Pattern Recognition", Rochelle Park, NJ: Hayden Book Co., Ch. 4.

[7]. Chow C. K. \& Liu C. N. (1968), "Approximating discrete probability distributions with dependence trees", IEEE Trans. Inform. Theory, vol. IT-14, no. 3, pp. 462-467.

[8].De Luca A. \& Termini S. (1972), “A Definition of a Non-probabilistic Entropy in Setting of Fuzzy Sets", Information and Control, 20, 301-312.

[9].Fan J. \& Xie W. (1999), "Distance Measures and Induced Fuzzy Entropy", Fuzzy Sets and Systems, 104, 305-314.

[10]. Gokhale D. V. \& Kullback S. (1978), "Information in Contingency Tables". New York: Marcel Dekker.

[11]. Gupta P., Arora H. D. \& Tiwari P. (2014). "A Measure of Divergence Between Fuzzy Sets With Advancement in Information Theory", International
Journal of Computer Applications, 118121.

[12]. Gupta P. \& Kumari S. (2014) “On Bounds for Weighted Fuzzy Mean Difference-Divergence Measures", International Journal of Scientific Research, vol. 3, Issue 6, 19-29.

[13]. Kadota T. T. \& Shepp L. A. (1967), "On the Best Finite Set of Linear Observables for Discriminating Two Gaussians Signals", IEEE Truns. Inform. Theory, vol. IT-13, no. 2, pp. 278-284.

[14]. Kailath T. (1967), "The Divergence and Bhattacharyya Distance Measures in Signal Selection", IEEE Transactions Commun. Technol., vol. COM-15, no. 1, pp. 52-60.

[15]. Kapur J.N. (1997), "Measures of Fuzzy Information", Mathematical Sciences trust Society, New Delhi.

[16]. Kazakos D. \& Cotsidas T. (1980), "A Decision Theory Approach to the Approximation of Discrete Probability Densities", IEEE Trans. Pattern Anal. Machine Intell., vol. PAMI-2, vol. 1, pp. 61-67.

[17]. Kullback S. (1968), "Information Theory and Statistics", New York: Dover Publications,

[18]. Kullback S. and Leibler R. A. (1951), "On Information and Sufficiency”, Ann. Math. Statistics, vol. 22, pp. 79-86.

[19]. Kumar T., Bajaj R. K. \& Gupta N. (2011), "On Some Parametric Generalized Measures of Fuzzy Information, Directed Divergence and Information Improvement", International Journal of Computer Applications, Vol. 30(9), pp. 5-10.

[20]. Lin J. \& Wong S. K. M. (1988), "Approximation of discrete probability distributions based on a new divergence measure", Congressus Numerantiitm, vol. 61, pp. 75-80. 
[21]. Lin J. (1991), “Divergence Measures Based on Shannon Entropy", IEEE Transactions

on Information Theory, Vol. 37(1), pp. 145-151.

[22]. Montes S., Couso I., Gil P. and C. Bertoluzza (2002), "Divergence Measure between Fuzzy Sets", International Journal of Approximate Reasoning, 30, 91-105.

[23]. Pal N. R. \& Bezdek J. C. (1994), "Measuring Fuzzy Uncertanity". IEEE Transactions on Fuzzy Systems, 2(2), 107-118.

[24]. Ohlan A. (2015), “A New Generalized Fuzzy Divergence Measure and Applications", Fuzzy Information Engineering, Vol. 7, pp. 507-523.

[25]. Ohlan A. \& Ohlan R.

"Generalizations of Fuzzy Information

Measures" , Springer Science and

Business Media LLC, 2016.

[26]. Parkash O. (2000), "On Fuzzy Symmetric Divergence", The Fourth Asian Fuzzy System Symposium, 2, 904-908.

[27]. Rani A., Sharma O. \& Gupta P.(2020), "Logarithmic Information Measure for Fuzzy Soft Matrices", International Journal of Statistics and Reliability Engineering",Vol. 7, no.1.

[28]. Rosenfeld A. (1985), "Distance Between Fuzzy Sets", Pattern Recognition Letters, 3, 229-231.

[29]. Shang X. \& Jiang, W. (1997), “A Note on Fuzzy Information Measures", Pattern

Recognition Letters, Vol. 18(5), pp. 425432.

[30]. Sharma O. (2018), "Fuzzy Soft Matrices Entropy: Application in DataReduction", International Journal of Fuzzy System Applications, vol.7, 5678.

[31]. Sharma O., Tiwari P., Gupta P. (2020), “ A New Non-Probabilistic
Divergence Measure of Fuzzy Matrix \& its Application", Songklanakarian Journal of Science and Technology, [32].

Vlachos I, Sergiadis G.D (2007), "Intuitionistic Fuzzy Information: Applications to Pattern Recognition", Pattern Recogn Lett 28(2):197-206.

[33]. Ye J. (2011), “Cosine Similarity Measure for Intuitionistic Fuzzy Sets and their Applications", Mathematical and Computer Modeling, 53, 91-97.

[34]. Zadeh L.A. (1965), "Fuzzy sets", Information and Control, 8, 338353.

\section{Creative Commons Attribution License 4.0} (Attribution 4.0 International, CC BY 4.0)

This article is published under the terms of the Creative Commons Attribution License 4.0 https://creativecommons.org/licenses/by/4.0/deed.en_US 